\title{
Direct evidence for close proximity of catalytic and regulatory domains of heterodimeric SGC based on fluorescence resonance energy transfer
}

\author{
Tobias Haase*, Nadine Haase and Soenke Behrends
}

Address: Institut für Pharmakologie, Toxikologie und Klinische Pharmazie, TU Braunschweig

Email: Tobias Haase* - tobias.haase@tu-bs.de

* Corresponding author

from 4th International Conference of cGMP Generators, Effectors and Therapeutic Implications

Regensburg, Germany. 19-2I June 2009

Published: II August 2009

BMC Pharmacology 2009, 9(SuppI I):P2I doi:I0.II86/I47I-22I0-9-SI-P2I

This abstract is available from: http://www.biomedcentral.com/I47I-22I0/9/SI/P2 I

(c) 2009 Haase et al; licensee BioMed Central Ltd.

\section{Background}

To examine the structural organisation of heterodimeric soluble guanylyl cyclase (sGC) fluorescence resonance energy transfer (FRET) was used to estimate distances between fluorescent proteins fused to the amino- and carboxy-terminal ends of the sGC $\beta_{1}$ and $\alpha$ subunits.

\section{Methods}

The FRET donor, CFP, and FRET acceptor, YFP, were fused to amino- and carboxy-terminal ends of sGC subunits. After generation of recombinant baculovirus strains fluorescent tagged sGC subunits were co-expressed in Sf9cells. Fluorescent variants of sGC were analyzed in vitro in cytosolic fractions by sensitized emission FRET. In addition, fluorescent tagged sGC subunits were analyzed in vivo using confocal laser scanning microscopy and fluorescence lifetime imaging (FLIM) on an inverted microscope.

\section{Results}

Carboxy-terminal fluorescent-tagged sGC combinations displayed NO stimulated sGC activity similar to the nontagged sGC heterodimer and showed in vitro and in vivo FRET values significantly higher than the negative control. Co-expression of amino-terminally tagged sGC showed also FRET. However, the enzyme complexes showed only basal enzyme activity. Co-expression of carboxy-terminally tagged $\alpha$ subunit with amino-terminally tagged $\beta_{1}$ subunit yielded a basally active enzyme complex that showed FRET. Co-expression of the amino-terminally tagged $\alpha$ subunit with the carboxy-terminally tagged $\beta_{1}$ subunit resulted an enzyme complex that showed NO stimulated activity and FRET.

\section{Discussion}

Based on the ability of an amino-terminal construct of the $\beta_{1}$ subunit (HNOX) to inhibit activity of an heterodimer consisting only of the catalytic domains $\left(\alpha_{\text {cat }} \beta_{\text {cat }}\right)$, Winger and Marletta [1] have proposed a direct interaction of the amino-terminal region of $\beta_{1}$ with the catalytic domains. Our results provide direct evidence that all four subunit termini of the heterodimeric enzyme complex are in proximity to each other. This supports the concept that sGC is structurally organized in a way that allows for direct interaction of the amino-terminal (HNOX) domains of $\beta_{1}$ (and $\alpha$ ) with the carboxy-terminal catalytic region.

\section{References}

I. Winger JA, Marletta MA: Expression and characterization of the catalytic domains of soluble guanylate cyclase: interaction with the heme domain. Biochemistry 2005, 44:4083-90. 